\title{
Clinical Trial of Fluid Infusion Rates for Pediatric Diabetic Ketoacidosis
}

\author{
Nathan Kuppermann, M.D., M.P.H., Simona Ghetti, Ph.D., Jeff E. Schunk, M.D., Michael J. \\ Stoner, M.D., Arleta Rewers, M.D., Ph.D., Julie K. McManemy, M.D., M.P.H., Sage R. Myers, \\ M.D., M.S.C.E, Lise E. Nigrovic, M.D., M.P.H., Aris Garro, M.D., M.P.H., Kathleen M. Brown, \\ M.D., Kimberly S. Quayle, M.D., Jennifer L. Trainor, M.D., Leah Tzimenatos, M.D., Jonathan \\ E. Bennett, M.D., Andrew D. DePiero, M.D., Maria Y. Kwok, M.D., M.P.H., Clinton S. Perry III, \\ Ph.D., Cody S. Olsen, M.S., T. Charles Casper, Ph.D., J. Michael Dean, M.D., and Nicole S. \\ Glaser, M.D. for the PECARN DKA FLUID Study Group*
}

\begin{abstract}
BACKGROUND—Diabetic ketoacidosis in children may cause brain injuries ranging from mild to severe. Whether intravenous fluids contribute to these injuries has been debated for decades.

METHODS-We conducted a 13-center, randomized, controlled trial that examined the effects of the rate of administration and the sodium chloride content of intravenous fluids on neurologic outcomes in children with diabetic ketoacidosis. Children were randomly assigned to one of four treatment groups in a 2 -by-2 factorial design $(0.9 \%$ or $0.45 \%$ sodium chloride content and rapid or slow rate of administration). The primary outcome was a decline in mental status (two consecutive Glasgow Coma Scale scores of $<14$, on a scale ranging from 3 to 15, with lower scores indicating worse mental status) during treatment for diabetic ketoacidosis. Secondary outcomes included clinically apparent brain injury during treatment for diabetic ketoacidosis, short-term memory during treatment for diabetic ketoacidosis, and memory and IQ 2 to 6 months after recovery from diabetic ketoacidosis.
\end{abstract}

RESULTS-A total of 1389 episodes of diabetic ketoacidosis were reported in 1255 children. The Glasgow Coma Scale score declined to less than 14 in 48 episodes (3.5\%), and clinically apparent brain injury occurred in 12 episodes $(0.9 \%)$. No significant differences among the treatment groups were observed with respect to the percentage of episodes in which the Glasgow Coma Scale score declined to below 14, the magnitude of decline in the Glasgow Coma Scale score, or the duration of time in which the Glasgow Coma Scale score was less than 14; with respect to the results of the tests of short-term memory; or with respect to the incidence of

\footnotetext{
Address reprint requests to Dr. Kuppermann at the Department of Emergency Medicine, University of California, Davis, School of Medicine, 2315 Stockton Blvd., Sacramento, CA 95817, or at nkuppermann@ucdavis.edu.

Additional members of the PECARN DKA FLUID Study Group are listed in the acknowledgments.

The authors' affiliations are listed in the Appendix.

The content and conclusions of this article are those of the authors and should not be construed as the official position or policy of, nor should any endorsements be inferred by, the Health Resources and Services Administration, the Department of Health and Human Services, or the U.S. government.

No potential conflict of interest relevant to this article was reported.

Disclosure forms provided by the authors are available with the full text of this article at NEJM.org.
} 
clinically apparent brain injury during treatment for diabetic ketoacidosis. Memory and IQ scores obtained after the children's recovery from diabetic ketoacidosis also did not differ significantly among the groups. Serious adverse events other than altered mental status were rare and occurred with similar frequency in all treatment groups.

CONCLUSIONS-Neither the rate of administration nor the sodium chloride content of intravenous fluids significantly influenced neurologic outcomes in children with diabetic ketoacidosis. (Funded by the Eunice Kennedy Shriver National Institute of Child Health and Human Development and the Health Resources and Services Administration; PECARN DKA FLUID ClinicalTrials.gov number, NCT00629707.)

Clinically apparent brain injuries occur in 0.5 to $0.9 \%$ of episodes of diabetic ketoacidosis in children; these brain injuries manifest as sudden neurologic decline and are often associated with morbidity and mortality. ${ }^{1-3}$ Among patients without obvious neurologic decline during treatment for diabetic ketoacidosis, subtle neurologic alterations are often present after recovery, including deficits in memory, attention, and $\mathrm{IQ}^{4-7}$ and changes in cerebral microstructure. ${ }^{4,8,9}$

Early theories to explain diabetic ketoacidosis-related brain injury suggested that rapid administration of intravenous fluids reduces serum osmolality, which results in brain swelling. ${ }^{10,11}$ Therefore, many treatment protocols for diabetic ketoacidosis in children advocate slow rehydration with isotonic fluids. Retrospective reviews have been used to support these strategies; however, those studies are subject to bias owing to the higher rates of brain injury that have been seen in children who are more dehydrated ${ }^{2}$ and therefore receive larger volumes of fluid. In addition, such studies have often included patients who had been selectively referred because of the severity of their illness from facilities that were often not specifically pediatric centers and that used treatment protocols that differed from those at pediatric centers. ${ }^{10,12,13}$ Rates of clinically apparent brain injury have remained stable over time, $, 2,14,15$ and properly controlled retrospective studies have not shown associations between the fluid administration rate and brain injury. ${ }^{2,3}$ Instead, data suggest that brain injury may result from abnormalities in cerebral perfusion and inflammation that occur during episodes of diabetic ketoacidosis. ${ }^{2,16}$ In the current trial, we investigated the effects of specific intravenous fluid regimens on neurologic outcomes in children with diabetic ketoacidosis.

\section{METHODS}

\section{OVERVIEW OF THE TRIAL}

We conducted this randomized, controlled trial at 13 emergency departments in the Pediatric Emergency Care Applied Research Network (PECARN), all of which were located in urban centers in the United States. We used a 2-by-2 factorial design to compare four rehydration treatment regimens in children with diabetic ketoacidosis (Table 1). ${ }^{17}$ We evaluated the effects of rehydration rate and fluid sodium chloride content on neurocognitive outcomes, including neurologic status during the episode of diabetic ketoacidosis and memory and IQ after recovery from diabetic ketoacidosis. Details of the trial methods were published 
previously ${ }^{17}$ and are also provided in the protocol, available with the full text of this article at NEJM.org.

\section{TRIAL OVERSIGHT}

The trial was designed by the first and last authors (principal investigators), with input from site investigators; collaborators from the PECARN data coordinating center; the PECARN quality, safety, and regulatory affairs subcommittee; the PECARN protocol review and development subcommittee; and the PECARN grant writing and publications subcommittee. Research coordinators collected the data under the supervision of the site investigators. The PECARN data coordinating center was responsible for data quality control and analyses. A data and safety monitoring board, which oversaw the conduct of the trial, included an expert in each of the following disciplines: emergency medicine, pediatric critical care, pediatric endocrinology, neuropsychology, and biostatistics. The data and safety monitoring board convened before enrollment, at the time of three scheduled interim efficacy analyses, and at the time of four additional scheduled reviews of safety, enrollment, and follow-up data. The authors vouch for the accuracy and completeness of the data and analyses and for the fidelity of the trial to the protocol. There was no industry funding for this trial, and there were no agreements concerning confidentiality of the data between the sponsor and the authors or institutions. All the authors reviewed drafts of the manuscript and agreed with the decision to submit the manuscript for publication.

\section{PATIENTS}

Children were eligible for enrollment in the trial if they were between 0 and 18 years of age and had received a diagnosis of diabetic ketoacidosis (defined as a blood glucose level of $>300 \mathrm{mg}$ per deciliter [16.7 mmol per liter] and either a venous $\mathrm{pH}$ of $<7.25$ or a serum bicarbonate level of $<15 \mathrm{mmol}$ per liter). Key exclusion criteria ${ }^{17}$ were underlying disorders that could affect mental status testing or neurocognitive evaluation; concurrent alcohol or narcotics use, head trauma, or other conditions that could affect neurologic function; diabetic ketoacidosis for which the patient had already received substantial treatment; known pregnancy; or factors for which treating physicians determined that a specific fluid and electrolyte therapy was necessary. Children who presented with a Glasgow Coma Scale score of 11 or lower (on a scale ranging from 3 to 15, with lower scores indicating worse mental status) were excluded after year 2 because many participating clinicians believed that fluid regimens for such children should not be determined on the basis of randomization.

\section{TREATMENTS}

Written informed consent was obtained from the parents or guardians of all enrolled patients. Assent was obtained from patients whose age met the minimum age for assent according to their local institutional review board. Children were then randomly assigned to one of four treatment regimens: fast rate of rehydration with fluid that had $0.45 \%$ sodium chloride content, fast rate of rehydration with fluid that had $0.9 \%$ sodium chloride content, slow rate of rehydration with fluid that had $0.45 \%$ sodium chloride content, and slow rate of rehydration with fluid that had $0.9 \%$ sodium chloride content. Details of the treatment regimens are provided in Table 1. Randomization was stratified according to baseline Glasgow Coma Scale score (14 or 15 vs. <14) and center (if the Glasgow Coma Scale score 
was <14). ${ }^{17}$ To avoid excessive restriction of the population available for enrollment, patients who had previously undergone randomization and subsequently had another episode of diabetic ketoacidosis during the trial were eligible to undergo randomization a second time. A patient could undergo randomization no more than twice. Additional details are provided in the Statistical Analysis section and in the protocol.

Treatment for diabetic ketoacidosis other than the rate of administration and the sodium chloride content of the fluid was identical in the four treatment groups. ${ }^{17}$ After administration of intravenous fluid boluses, insulin treatment was initiated as a continuous intravenous infusion at a rate of $0.1 \mathrm{U}$ per kilogram of body weight per hour. To prevent hypoglycemia during insulin treatment, dextrose was added to the study fluids when the serum glucose level declined below 200 to $300 \mathrm{mg}$ per deciliter (11.1 to $16.7 \mathrm{mmol}$ per liter). Patients and their parents or guardians were unaware of the treatment-group assignments. It was not possible for clinicians to be unaware of the treatment-group assignments because of the need to know the fluid protocol for clinical decision making.

\section{OUTCOMES}

The primary trial outcome was deterioration of neurologic status (as evidenced by two consecutive Glasgow Coma Scale scores of $<14$ during any hour within the first 24 hours of treatment for diabetic ketoacidosis). Secondary outcomes included short-term memory during treatment for diabetic ketoacidosis (forward and backward digit-span recall; scores range from 0 to 16 , with higher scores indicating better short-term memory) ${ }^{18}$; clinically apparent brain injury (defined as a deterioration in neurologic status leading to initiation of hyperosmolar therapy or endotracheal intubation or resulting in death) during treatment for diabetic ketoacidosis; and short-term memory, contextual memory, and IQ 2 to 6 months after the episode of diabetic ketoacidosis. In the digit span test, participants are asked to repeat a sequence of numbers presented orally. In the "forward" task, participants are asked to repeat numbers in order, as presented. In the "backward" task, participants are asked to list the numbers in reverse order. The test stops when participants report the incorrect sequence twice for a given digit-span length.

To address variations in the diagnosis of clinically apparent brain injury, records of encounters with patients in which hyperosmolar therapy, endotracheal intubation, or death were documented were reviewed by an adjudication committee that included two pediatric critical care physicians and one pediatric emergency medicine physician, all of whom were unaware of the treatment-group assignments. Committee members confirmed or rejected each diagnosis of clinically apparent brain injury on the basis of published criteria. ${ }^{19}$

\section{ASSESSMENTS}

Assessments of Mental Status-Glasgow Coma Scale scores were assessed at enrollment and hourly thereafter. Glasgow Coma Scale scores of less than 14 were confirmed by repeating the test 15 minutes later. For children 3 years of age or older, digitspan tests were conducted at enrollment and every 4 hours thereafter during normal waking hours. Glasgow Coma Scale and digit-span assessments continued for 24 hours or until 
resolution of diabetic ketoacidosis (as defined by the transition to subcutaneous insulin) if diabetic ketoacidosis resolved before the 24-hour time point.

Assessments of Memory Function and IQ-Patients 3 to 18 years of age were asked to return 2 to 4 months after discharge from the hospital for neurocognitive assessment but were allowed to return up to 6 months after discharge. Neurocognitive testing was rescheduled in the event of either hypoglycemia (defined as a glucose level of $<70 \mathrm{mg}$ per deciliter [3.9 mmol per liter]) or ketosis (defined as the presence of moderate or large urine ketones).

IQ was evaluated with the use of the Wechsler Abbreviated Scale of Intelligence ${ }^{20}$ (in patients 6 years of age or older) and the Wechsler Preschool and Primary Scale of Intelligence short form (in patients 3 to 5 years of age). ${ }^{21}$ At the same testing session, the digit-span test was repeated, and contextual memory was assessed with color and spatialposition tasks, ${ }^{17}$ which evaluated item recognition and recollection of contextual detail. Shorter and simpler versions of these tasks were used for children 3 to 5 years of age. ${ }^{17}$ Research personnel who conducted the cognitive testing and recorded outcome data were unaware of the treatment-group assignments, as were the trial investigators who oversaw this process.

\section{STATISTICAL ANALYSIS}

The primary analyses were performed according to the intention-to-treat principle. We also performed secondary analyses in the per-protocol population (which included patients who underwent randomization and received trial fluids per the protocol) and in the safety population (which included all patients who received any trial fluid) (details are provided in the protocol). Patients who had Glasgow Coma Scale scores of 14 or 15 at baseline were included in the primary analyses. Because patients who had Glasgow Coma Scale scores of less than 14 at baseline had already met the criterion for the primary outcome before enrollment, such patients were not included in the primary analyses but were included in the secondary analyses. Distinct encounters with the same patient were considered to be independent events. Given that patients who underwent randomization a second time could have been randomly assigned to a treatment regimen that was different from the first regimen they had been assigned to, a single patient could be represented in more than one treatment group in the analyses. Cochran-Mantel-Haenszel tests were used to test the effects of the rate of administration and of the sodium chloride content of the fluid. Each of these factors was tested with the use of $\mathrm{P}$ values that were adjusted for multiplicity at a twosided alpha level of 0.025 .

We analyzed the magnitude of the decline in the Glasgow Coma Scale score and the duration of time in which the Glasgow Coma Scale score was less than 14 using Van Elteren tests, with adjustment for stratification variables. The incidence of clinically apparent brain injury was evaluated with the use of a Cochran-Mantel-Haenszel test. We tested for treatment interactions using regression models. To evaluate digit-span scores, we used a linear mixedeffects model to estimate time-dependent effects of the rate of administration and the sodium chloride content of the fluid. We assigned a digit-span score of zero in cases in which a 
patient had a Glasgow Coma Scale score of less than 14 to account for digit-span scores that could not be measured owing to mental status alterations. A random intercept and slope term for patient encounters and similar fixed terms for trial centers were included in the model.

The main analysis of memory function was based on the average of the children's correct recall rates of items in association with their color background or spatial location. Scores were excluded from the analysis if chance recognition of previously viewed items was observed (sensitivity index of $<0.50 ; 5 \%$ of tests). Memory scores and IQ were compared with the use of a Van Elteren test, with adjustment for stratification variables. In cases in which a patient had more than one valid follow-up measurement, only the first measurement was included in the analysis. Significance levels for digit-span score and memory function were adjusted for multiple comparisons with the use of the Holm procedure.

We also analyzed treatment effects in pre-specified subgroups defined according to age $(<6$ years vs. $\ 6$ years), Glasgow Coma Scale score at baseline, and history of diabetic ketoacidosis (yes vs. no) using regression models, with adjustment for stratification variables. The overall type I error rate for the subgroup analyses was preserved at 0.05 . Using the same methods, we explored treatment effects in eight subgroups defined according to various baseline characteristics that are associated with more severe diabetic ketoacidosis; results from four of these subgroups are reported and should be interpreted with caution owing to multiple comparisons. The analyses of statistical power and sample size have been described previously. ${ }^{17}$ Our target sample size was 1360 patient encounters involving children who had baseline Glasgow Coma Scale scores of 14 or 15 (i.e., 1360 episodes of diabetic ketoacidosis that could be included in the primary analysis).

Two-sided O'Brien-Fleming stopping boundaries at the time of each interim efficacy analysis were calculated with the use of the alpha-spending function approach. The thresholds for significance at the first, second, and third interim analyses were 0.000006 , 0.0008 , and 0.0075 , respectively. No safety concerns were identified, and the trial proceeded to completion.

\section{RESULTS}

\section{PATIENTS}

From February 2011 through September 2016, a total of 1255 children were randomly assigned to one of the four treatment regimens (Table 1). Among these 1255 patients, 132 had a second episode of diabetic ketoacidosis and underwent randomization a second time during the trial. Two additional patients who had a third episode of diabetic ketoacidosis inadvertently underwent randomization a third time. Therefore, a total of 1389 distinct episodes of diabetic ketoacidosis were evaluated. Patient enrollment and randomization status are shown in Figure 1. Demographic and clinical characteristics of the children, including status with respect to history of diabetes, did not differ significantly among the four groups (Table 2). Adherence to the assigned treatment regimens was excellent (Figs. S1 and S2 in the Supplementary Appendix, available at NEJM.org). Enrollment varied among the emergency departments; the number of patient encounters ranged from 23 to 239 (Table S1 in the Supplementary Appendix). 


\section{MENTAL STATUS DURING TREATMENT FOR DIABETIC KETOACIDOSIS}

In 1361 episodes of diabetic ketoacidosis (98.0\%), children presented with Glasgow Coma Scale scores of 14 or 15, and these episodes were therefore included in the primary analysis. During the course of the trial, the Glasgow Coma Scale score declined to below 14 in 48 (3.5\%) of these episodes (Table 3). A total of 22 episodes (1.6\%) resulted in the children receiving hyperosmolar therapy for possible cerebral edema or brain injury. In 12 episodes $(0.9 \%)$, the children had clinically apparent brain injury (confirmed by adjudication) - a rate that was similar to previously documented frequencies. ${ }^{1-3}$ Most of the 12 patients presented with severe acidosis and hypocapnia (Table S2 in the Supplementary Appendix). One of the 12 patients died, and the remaining patients recovered without overt neurologic deficits.

There were no significant differences among the groups in the percentage of episodes in which the Glasgow Coma Scale score declined to below 14, in the magnitude of decline in the Glasgow Coma Scale score, or in the duration of time in which the Glasgow Coma Scale score was less than 14 (Table 3, and Table S3 in the Supplementary Appendix). The Breslow-Day test for homogeneity of the odds ratios did not provide evidence against homogeneity $(\mathrm{P}=0.39$ for administration rate; $\mathrm{P}=0.67$ for sodium chloride content). The incidence of clinically apparent brain injury was higher in the slow rehydration groups than in the fast rehydration groups; however, the differences were not significant. Digit-span scores during the episode of diabetic ketoacidosis did not differ significantly among the four groups, although point estimates for the rate of improvement in forward digit-span scores favored more rapid rehydration $(\mathrm{P}=0.06)$.

\section{SUBGROUP ANALYSES OF MENTAL STATUS}

Analyses of the relative risk of a decline to below 14 in the Glasgow Coma Scale score in subgroups defined according to age and history of diabetic ketoacidosis among patients who had Glasgow Coma Scale scores of 14 or 15 at baseline did not show differential treatment effects (Fig. S3 in the Supplementary Appendix). In the subgroups of patients who had more severe diabetic ketoacidosis (a pH or partial pressure of carbon dioxide $\left[\mathrm{P}_{\mathrm{CO}_{2}}\right]$ level in the lowest quartile or a blood urea nitrogen level or glucose level in the highest quartile), the percentage of episodes in which the Glasgow Coma Scale score declined to below 14 and the percentage of episodes in which clinically apparent brain injury was confirmed did not differ significantly among the groups (Table S4 in the Supplementary Appendix). The effect of rehydration rate on forward digit-span scores differed in the subgroup defined according to $\mathrm{P}_{\mathrm{Co}_{2}}$ level $\left(\mathrm{P}=0.03\right.$ for the interaction between treatment and $\mathrm{P}_{\mathrm{Co}_{2}}$ level), with faster improvement in the rapid rehydration groups than in the slow rehydration groups among patients with a low $\mathrm{P}_{\mathrm{CO}_{2}}$ level $(\mathrm{P}=0.03)$. The effect of rehydration rate on backward digitspan scores differed in the subgroup defined according to $\mathrm{pH}$ level $(\mathrm{P}=0.01$ for the interaction between treatment and $\mathrm{pH}$ level), with faster improvement in the rapid rehydration groups than in the slow rehydration groups among patients with a low $\mathrm{pH}(\mathrm{P}=$ $0.01)$. 


\section{NEUROCOGNITIVE ASSESSMENTS AFTER RECOVERY FROM DIABETIC KETOACIDOSIS}

In all, 1287 episodes occurred in children older than 3 years of age who met the criteria for follow-up neurocognitive testing. The children involved in 387 of these episodes (30.1\%) were either lost to follow-up or declined to return for neurocognitive testing (see "Power and Sample Size" in the Supplementary Appendix for sensitivity analyses regarding missing data). Data were analyzed for 855 episodes (66.4\%), for which follow-up occurred within 6 months after the patient's discharge from the hospital ( 756 within 4 months and 99 between 5 and 6 months). There were no significant differences in neurocognitive outcomes after recovery among the trial groups (Table S5 in the Supplementary Appendix).

\section{RESULTS AMONG PATIENTS TREATED ACCORDING TO THE PROTOCOL}

Fluid hydration regimens that were administered in 115 episodes (8.3\%), including 107 (7.9\%) analyzed for the primary outcome, deviated sufficiently from the assigned treatment regimen that the rate of administration or sodium chloride content of the fluid was more similar to other treatment regimens than to the assigned regimen. We excluded these episodes and repeated the analyses to determine whether these protocol deviations influenced the outcomes (Tables S6 and S7 in the Supplementary Appendix). The results of these analyses showed no significant differences among the groups.

\section{RESULTS AMONG PATIENTS ACCORDING TO TREATMENT RECEIVED}

We modeled continuous versions of fluid administration rate and sodium chloride content in regression analyses. To account for influences of clinical presentation on decisions of the clinicians to deviate from assigned treatment regimens, we included covariates that reflected the severity of diabetic ketoacidosis (levels of $\mathrm{pH}, \mathrm{P}_{\mathrm{co}_{2}}$, glucose, sodium, and blood urea nitrogen), age, and whether the diabetes was new-onset or preexisting. Again, no significant effects of either fluid administration rate or sodium chloride content on acute neurologic outcomes were found (data not shown).

\section{NONNEUROLOGIC ADVERSE EVENTS}

Hyperchloremic acidosis was more common among patients who received fluid that had a $0.9 \%$ sodium chloride content than among those who received fluid that had a $0.45 \%$ sodium chloride content and more common among patients who received fluid at a rapid rate than among those who received fluid at a slow rate (Table S8 in the Supplementary Appendix). The $0.9 \%$ sodium chloride regimens were also associated with a higher incidence of hypocalcemia and hypophosphatemia than were the $0.45 \%$ sodium chloride regimens. A rapid rate of fluid administration was associated with a higher incidence of hypocalcemia but not a higher incidence of hypophosphatemia. Hypoglycemia and hypokalemia occurred at similar rates in the four groups. Serious adverse events occurred in less than $3 \%$ of participants (Table 4, and Table S9 in the Supplementary Appendix). The time to resolution of diabetic ketoacidosis and the duration of hospitalization were similar among the groups (Table S10 in the Supplementary Appendix). 


\section{DISCUSSION}

In this randomized, controlled trial, with a 2-by-2 factorial design, that involved children with diabetic ketoacidosis, there were no significant differences in the rate of decline in mental status or in the rate of clinically apparent brain injury during treatment for diabetic ketoacidosis or in neurocognitive function after recovery from diabetic ketoacidosis among patients who received rehydration fluid at two different administration rates and with two different sodium chloride contents. The lowest rates of mental status decline and clinically apparent brain injury were in the rapid-rehydration groups, although the differences from other groups were not significant. Furthermore, analyses of the subgroups of patients who had more severe diabetic ketoacidosis suggested faster improvement in digit-span recall in the rapid fluid-administration groups than in the slow fluid-administration groups. These findings underscore the lack of a causal association between rapid fluid administration and diabetic ketoacidosis-related brain injury.

Clinically apparent diabetic ketoacidosis-related brain injury occurs infrequently but is an important cause of neurologic damage and death among children with diabetes. ${ }^{1-3,22-24}$ Subtle brain injury often occurs during treatment for diabetic ketoacidosis in children and may contribute to cognitive decline. ${ }^{4-9}$ Excessive fluid administration, which may result in rapid osmotic changes, has been widely suspected to cause brain injury, ${ }^{11,25,26}$ but a more recent hypothesis suggests that cerebral hypoperfusion and the effects of reperfusion, along with neuroinflammation, are central to diabetic ketoacidosis-related brain injury. ${ }^{2,27-29}$ The latter hypothesis is consistent with reports that document symptomatic and even fatal brain injury occurring before the initiation of treatment for diabetic ketoacidosis. ${ }^{2,30}$ Furthermore, although cerebral edema is a feature of clinically apparent brain injury, edema often develops hours or days after a diagnosis of brain injury, ${ }^{19}$ a finding that suggests that edema may be a consequence, rather than the cause, of brain injury.

Studies involving children with diabetic ketoacidosis and studies in rodent models suggest similarities between diabetic ketoacidosis-related brain injury and ischemia-reperfusion injury. These include low cerebral blood flow and brain-cell swelling, along with low levels of high-energy phosphates in the brain and elevated lactate levels, during untreated episodes of diabetic ketoacidosis and cerebral hyperemia and vasogenic edema during treatment for diabetic ketoacidosis. ${ }^{27-29,31-33}$ Although alterations in cerebral blood flow may be involved in diabetic ketoacidosis-related brain injury, the severity of cerebral hypoperfusion is unlikely to be sufficient to cause brain injury in the absence of other contributing factors. Diabetic ketoacidosis is associated with marked systemic increases in inflammatory cytokines and chemokines that may contribute to brain injury by activating cerebrovascular endothelia and increasing leukocyte adhesion. ${ }^{16,34-36}$ Elevated levels of matrix metalloproteinase may promote blood-brain barrier dysfunction. ${ }^{37}$

The current trial has several limitations. First, the fluid administration rates were selected to represent upper and lower boundaries of current protocols used to treat pediatric diabetic ketoacidosis. It is possible that the use of administration rates outside this range may have resulted in different outcomes. However, given that the lowest rate of decline in mental status occurred in the group that received rapid rehydration with $0.45 \%$ sodium chloride 
solution, it seems unlikely that larger differences between protocols would have instead favored slower rehydration. Second, clinically apparent brain injury occurs in less than $1 \%$ of episodes, making it impractical to design a trial with sufficient statistical power to detect differences in this outcome. We used alterations in mental status as an indicator of subtle brain injury because children who have abnormal Glasgow Coma Scale scores during treatment for diabetic ketoacidosis are more likely to have subtle cerebral edema (on magnetic resonance imaging) than those who have normal mental status during treatment. 31,38 Nonetheless, it is possible that declines in Glasgow Coma Scale scores that occur during treatment for diabetic ketoacidosis reflect physiological processes that are different from those responsible for clinically apparent brain injury. Third, statistical power may have been reduced by the inclusion of repeat episodes, although such a reduction would be small.

In conclusion, in this prospective, randomized trial, neither the rate of administration nor the sodium chloride content of intravenous fluids significantly influenced neurologic outcomes of diabetic ketoacidosis in children.

\section{Supplementary Material}

Refer to Web version on PubMed Central for supplementary material.

\section{Acknowledgments}

Supported by the Eunice Kennedy Shriver National Institute of Child Health and Human Development (grant U01HD062417) and the Emergency Medical Services for Children Network Development Demonstration Program of the Maternal and Child Health Bureau, Health Resources and Services Administration, under cooperative agreement (awards U03MC00008, U03MC00001, U03MC00003, U03MC00006, U03MC00007, U03MC22684, and U03MC22685).

We thank Marci Fjelstad and Amy Watson from the PECARN Data Coordinating Center for their assistance; the research coordinators in PECARN, without whom this trial would not have been possible; the clinicians in PECARN who enrolled children into this trial; the members of the data and safety monitoring board (Roger Lewis, M.D., Ph.D., Jeffrey Blumer, M.D., Ph.D., Andrew Bremer, M.D., Ph.D., Thomas Cook, Ph.D., and Beth Slomine, Ph.D., A.B.P.P.); and the members of the study outcome adjudication committee (Kathleen Meert, M.D., Jerry Zimmerman, M.D., Ph.D., and Robert Hickey, M.D.).

\section{References}

1. Edge JA, Hawkins MM, Winter DL, Dunger DB. The risk and outcome of cerebral oedema developing during diabetic ketoacidosis. Arch Dis Child. 2001; 85:16-22. [PubMed: 11420189]

2. Glaser N, Barnett P, McCaslin I, et al. Risk factors for cerebral edema in children with diabetic ketoacidosis. N Engl J Med. 2001; 344:264-9. [PubMed: 11172153]

3. Lawrence SE, Cummings EA, Gaboury I, Daneman D. Population-based study of incidence and risk factors for cerebral edema in pediatric diabetic ketoacidosis. J Pediatr. 2005; 146:688-92. [PubMed: 15870676]

4. Cameron FJ, Scratch SE, Nadebaum C, et al. Neurological consequences of diabetic ketoacidosis at initial presentation of type 1 diabetes in a prospective cohort study of children. Diabetes Care. 2014; 37:1554-62. [PubMed: 24855156]

5. Cato MA, Mauras N, Mazaika P, et al. Longitudinal evaluation of cognitive functioning in young children with type 1 diabetes over 18 months. J Int Neuropsychol Soc. 2016; 22:293-302. [PubMed: 26786245]

6. Ghetti S, Lee JK, Sims CE, Demaster DM, Glaser NS. Diabetic ketoacidosis and memory dysfunction in children with type 1 diabetes. J Pediatr. 2010; 156:109-14. [PubMed: 19833353] 
7. Semenkovich K, Bischoff A, Doty T, et al. Clinical presentation and memory function in youth with type 1 diabetes. Pediatr Diabetes. 2016; 17:492-9. [PubMed: 26377697]

8. Antenor-Dorsey JA, Meyer E, Rutlin J, et al. White matter microstructural integrity in youth with type 1 diabetes. Diabetes. 2013; 62:581-9. [PubMed: 23139349]

9. Siller AF, Lugar H, Rutlin J, et al. Severity of clinical presentation in youth with type 1 diabetes is associated with differences in brain structure. Pediatr Diabetes. 2017; 18:686-95. [PubMed: 27488913]

10. Duck SC, Wyatt DT. Factors associated with brain herniation in the treatment of diabetic ketoacidosis. J Pediatr. 1988; 113:10-4. [PubMed: 3133455]

11. Harris GD, Fiordalisi I, Finberg L. Safe management of diabetic ketoacidemia. J Pediatr. 1988; 113:65-8. [PubMed: 3133458]

12. Harris GD, Fiordalisi I, Harris WL, Mosovich LL, Finberg L. Minimizing the risk of brain herniation during treatment of diabetic ketoacidemia: a retrospective and prospective study. $\mathbf{J}$ Pediatr. 1990; 117:22-31. [PubMed: 2115081]

13. White PC. Optimizing fluid management of diabetic ketoacidosis. Pediatr Diabetes. 2015; 16:3179. [PubMed: 25832987]

14. Mel JM, Werther GA. Incidence and outcome of diabetic cerebral oedema in childhood: are there predictors? J Paediatr Child Health. 1995; 31:17-20. [PubMed: 7748682]

15. Patel A, Singh D, Bhatt P, Thakkar B, Akingbola OA, Srivastav SK. Incidence, trends, and outcomes of cerebral edema among children with diabetic ketoacidosis in the United States. Clin Pediatr (Phila). 2016; 55:943-51. [PubMed: 26603587]

16. Close TE, Cepinskas G, Omatsu T, et al. Diabetic ketoacidosis elicits systemic inflammation associated with cerebrovascular endothelial cell dysfunction. Micro-circulation. 2013; 20:534-43.

17. Glaser NS, Ghetti S, Casper TC, Dean JM, Kuppermann N. Pediatric diabetic ketoacidosis, fluid therapy, and cerebral injury: the design of a factorial randomized controlled trial. Pediatr Diabetes. 2013; 14:435-46. [PubMed: 23490311]

18. Baddeley A. Gloucestershire United Kingdom: Clarendon Press; 1986Working memory.

19. Muir AB, Quisling RG, Yang MC, Rosenbloom AL. Cerebral edema in childhood diabetic ketoacidosis: natural history, radiographic findings, and early identification. Diabetes Care. 2004; 27:1541-6. [PubMed: 15220225]

20. Wechsler D. Wechsler abbreviated scale of intelligence San Antonio, TX: Psychological Corporation; 1999

21. Tsushima WT. Short form of the WPPSI and WPPSI-R. J Clin Psychol. 1994; 50:877-80. [PubMed: 7896922]

22. Daneman D. Diabetes-related mortality: a pediatrician's view. Diabetes Care. 2001; 24:801-2. [PubMed: 11347732]

23. Edge JA, Ford-Adams ME, Dunger DB. Causes of death in children with insulin dependent diabetes 1990-96. Arch Dis Child. 1999; 81:318-23. [PubMed: 10490436]

24. Scibilia J, Finegold D, Dorman J, Becker D, Drash A. Why do children with diabetes die? Acta Endocrinol Suppl (Copenh). 1986; 279:326-33. [PubMed: 3096039]

25. Arieff AI, Kleeman CR. Cerebral edema in diabetic comas. II. Effects of hyperosmolality, hyperglycemia and insulin in diabetic rabbits. J Clin Endocrinol Metab. 1974; 38:1057-67. [PubMed: 4831702]

26. Prockop LD. Hyperglycemia, polyol accumulation, and increased intracranial pressure. Arch Neurol. 1971; 25:126-40. [PubMed: 4998960]

27. Glaser NS, Wootton-Gorges SL, Marcin JP, et al. Mechanism of cerebral edema in children with diabetic ketoacidosis. J Pediatr. 2004; 145:164-71. [PubMed: 15289761]

28. Glaser N, Yuen N, Anderson SE, Tancredi DJ, O’Donnell ME. Cerebral metabolic alterations in rats with diabetic ketoacidosis: effects of treatment with insulin and intravenous fluids and effects of bumetanide. Diabetes. 2010; 59:702-9. [PubMed: 20028943]

29. Yuen N, Anderson SE, Glaser N, Tancredi DJ, O'Donnell ME. Cerebral blood flow and cerebral edema in rats with diabetic ketoacidosis. Diabetes. 2008; 57:2588-94. [PubMed: 18633109] 
30. Couch RM, Acott PD, Wong GW. Early onset fatal cerebral edema in diabetic ketoacidosis. Diabetes Care. 1991; 14:78-9.

31. Glaser NS, Marcin JP, Wootton-Gorges SL, et al. Correlation of clinical and biochemical findings with diabetic ketoacidosis-related cerebral edema in children using magnetic resonance diffusionweighted imaging. J Pediatr. 2008; 153:541-6. [PubMed: 18589447]

32. Glaser NS, Tancredi DJ, Marcin JP, et al. Cerebral hyperemia measured with near infrared spectroscopy during treatment of diabetic ketoacidosis in children. J Pediatr. 2013; 163:1111-6. [PubMed: 23871731]

33. Lam TI, Anderson SE, Glaser N, O’Donnell ME. Bumetanide reduces cerebral edema formation in rats with diabetic ketoacidosis. Diabetes. 2005; 54:510-6. [PubMed: 15677509]

34. Hoffman WH, Passmore GG, Hannon DW, et al. Increased systemic Th17 cytokines are associated with diastolic dysfunction in children and adolescents with diabetic ketoacidosis. PLoS One. 2013; 8(8):e71905. [PubMed: 24013901]

35. Omatsu T, Cepinskas G, Clarson C, et al. CXCL1/CXCL8 (GROa/IL-8) in human diabetic ketoacidosis plasma facilitates leukocyte recruitment to cerebrovascular endothelium in vitro. Am J Physiol Endocrinol Metab. 2014; 306(9):E1077-E1084. [PubMed: 24619879]

36. Stentz FB, Umpierrez GE, Cuervo R, Kitabchi AE. Proinflammatory cytokines, markers of cardiovascular risks, oxidative stress, and lipid peroxidation in patients with hyperglycemic crises. Diabetes. 2004; 53:2079-86. [PubMed: 15277389]

37. Garro A, Chodobski A, Szmydynger-Chodobska J, et al. Circulating matrix metalloproteinases in children with diabetic ketoacidosis. Pediatr Diabetes. 2017; 18:95-102. [PubMed: 26843101]

38. Glaser NS, Wootton-Gorges SL, Buonocore MH, et al. Frequency of subclinical cerebral edema in children with diabetic ketoacidosis. Pediatr Diabetes. 2006; 7:75-80. [PubMed: 16629712]

\section{Appendix}

The authors' affiliations are as follows: the Departments of Emergency Medicine (N.K., L.T.), Pediatrics (N.K., N.S.G.), and Psychology (S.G., C.S.P.), University of California Davis Health, University of California, Davis, School of Medicine, Sacramento; the Department of Pediatrics, University of Utah School of Medicine, Salt Lake City (J.E.S., C.S.O., T.C.C., J.M.D.); the Division of Emergency Medicine, Department of Pediatrics, Nationwide Children's Hospital, Ohio State University College of Medicine, Columbus (M.J.S.); the Division of Emergency Medicine, Department of Pediatrics, Colorado Children's Hospital, University of Colorado-Denver School of Medicine, Aurora (A.R.); the Division of Emergency Medicine, Department of Pediatrics, Texas Children's Hospital, Baylor College of Medicine, Houston (J.K.M.); the Division of Emergency Medicine, Department of Pediatrics, Children's Hospital of Philadelphia, Perelman School of Medicine at the University of Pennsylvania (S.R.M.), and the Division of Emergency Medicine, Nemours/A.I. duPont Hospital for Children, Sidney Kimmel Medical College at Thomas Jefferson University (J.E.B., A.D.D.) — both in Philadelphia; the Division of Emergency Medicine, Department of Pediatrics, Boston Children's Hospital, Harvard Medical School, Boston (L.E.N.); the Departments of Emergency Medicine and Pediatrics, Rhode Island Hospital, Warren Alpert Medical School of Brown University, Providence (A.G.); the Division of Emergency Medicine, Department of Pediatrics, Children's National Medical Center, George Washington School of Medicine and Health Sciences, Washington, DC (K.M.B.); the Division of Emergency Medicine, Department of Pediatrics, St. Louis Children's Hospital, Washington University School of Medicine in St. Louis, St. Louis (K.S.Q.); the Division of Emergency Medicine, Department of Pediatrics, Ann and Robert H. Lurie Children's Hospital of Chicago, Northwestern University Feinberg School of 
Medicine, Chicago (J.L.T.); and the Division of Emergency Medicine, Department of Pediatrics, New York Presbyterian Morgan Stanley Children's Hospital, Columbia University College of Physicians and Surgeons, New York (M.Y.K.); and the Department of Psychology, Tufts University, Medford, MA (C.S.P.). 


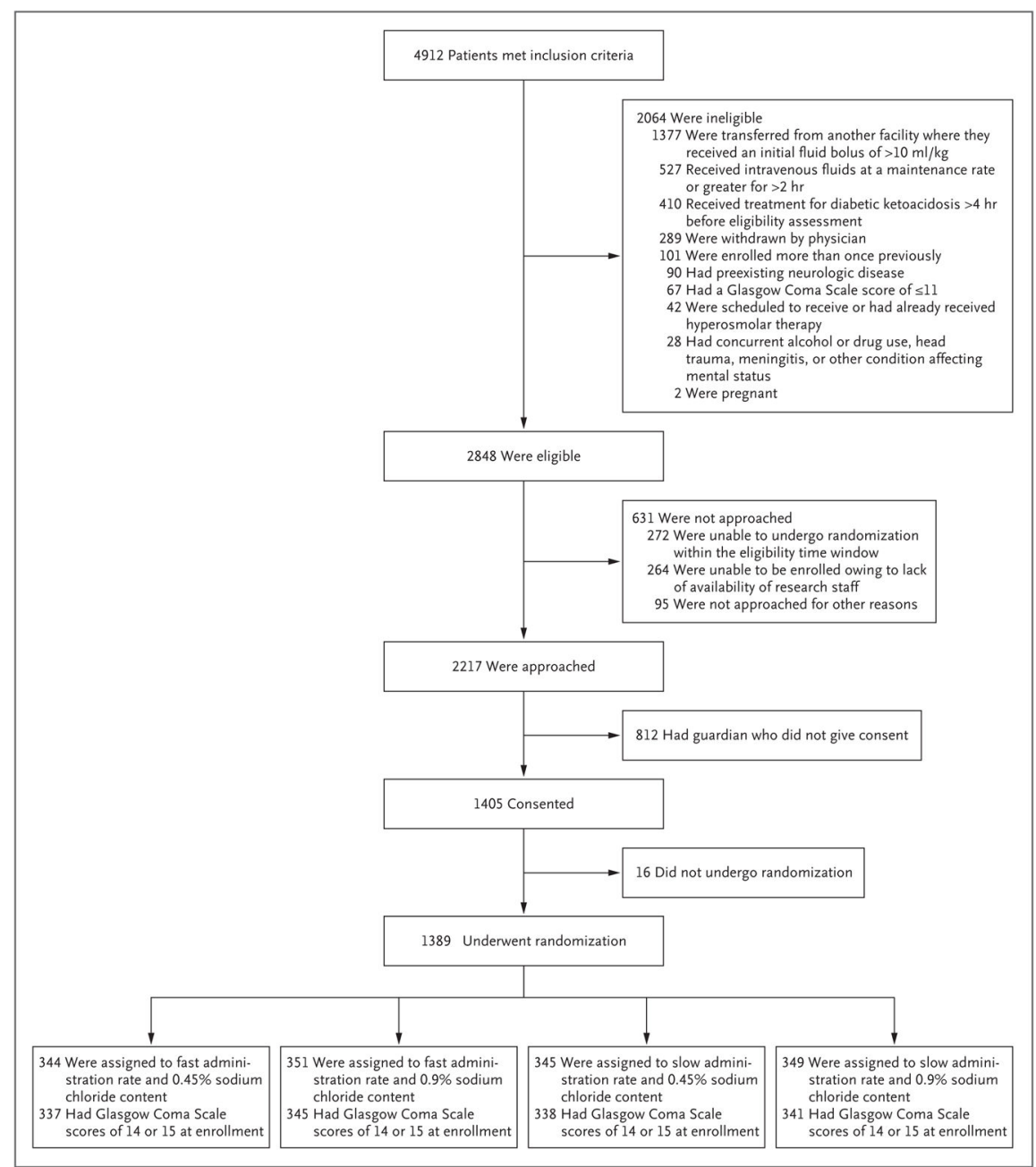

Figure 1. Enrollment and Randomization

A total of 586 patients met multiple ineligibility criteria. Three patients who had a Glasgow Coma Scale score of 11 or lower were enrolled before the implementation of this exclusion criterion. Patients were permitted to undergo randomization a second time if they had a second episode of diabetic ketoacidosis during the trial, with each randomization considered to be a distinct encounter; hence, some children are represented more than once in this figure. A total of 1255 patients underwent randomization initially. Among these 1255 patients, 132 had a second episode of diabetic ketoacidosis and underwent randomization a second time during the trial. Two additional patients who had a third episode of diabetic ketoacidosis inadvertently underwent randomization a third time. Therefore, a total of 1389 distinct episodes of diabetic ketoacidosis were evaluated, as shown here. Enrollment sites were located in Boston, MA, Chicago, IL, Columbus, OH, Denver, CO, Houston, TX, New York, NY, Philadelphia, PA, Providence, RI, Sacramento, CA, Salt Lake City, UT, St. Louis, MO, Washington, DC, and Wilmington, DE. 


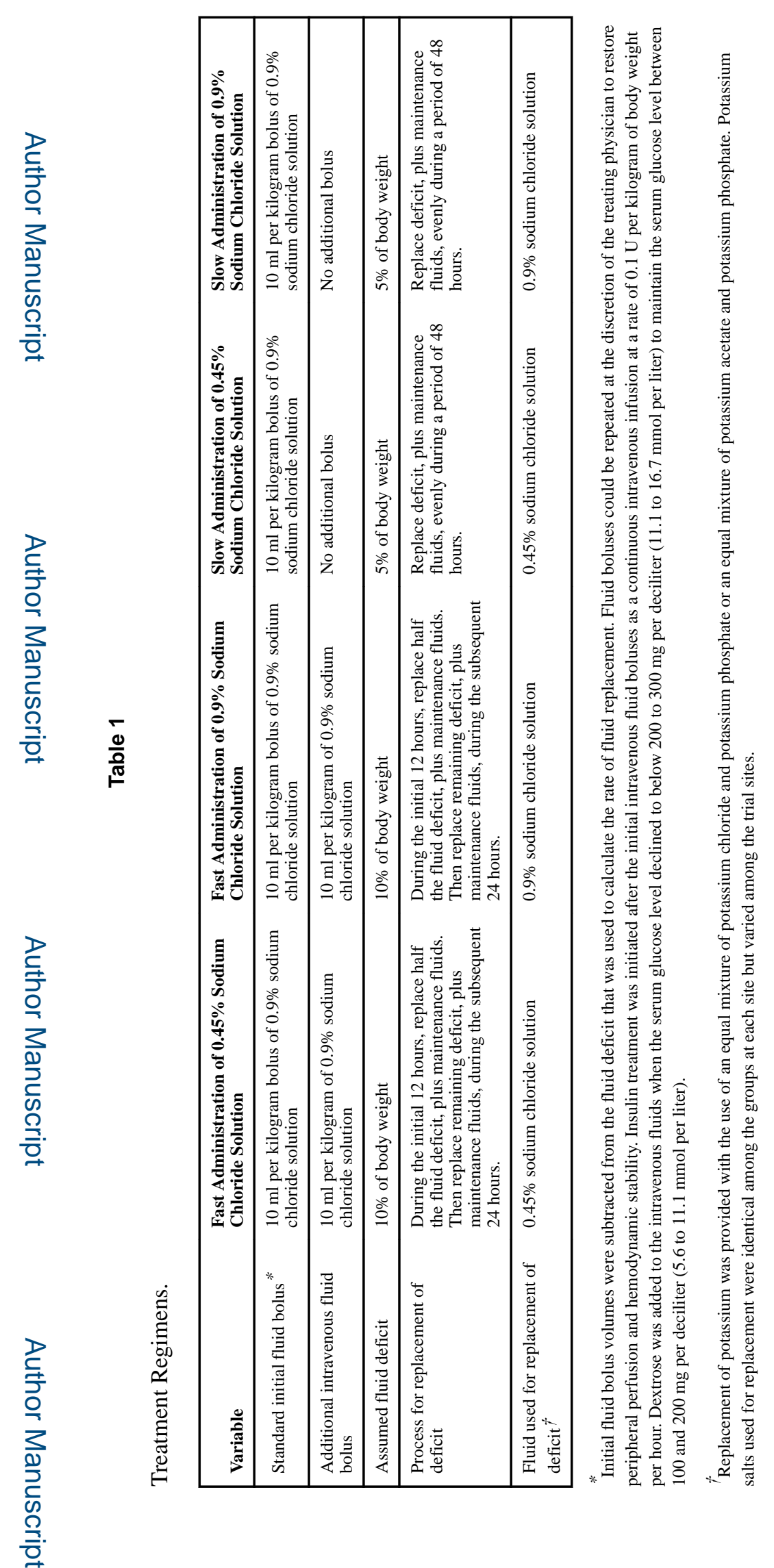

NEngl J Med. Author manuscript; available in PMC 2018 December 14. 
Table 2

Demographic and Clinical Characteristics of the Trial Population. *

\begin{tabular}{|c|c|c|c|c|}
\hline Variable & $\begin{array}{l}\text { Fast Administration } \\
\text { of 0.45\% Sodium } \\
\text { Chloride Solution (N } \\
=\mathbf{3 4 4})\end{array}$ & $\begin{array}{l}\text { Fast Administration } \\
\text { of } 0.9 \% \text { Sodium } \\
\text { Chloride Solution }(\mathbf{N} \\
\quad=351)\end{array}$ & $\begin{array}{l}\text { Slow Administration } \\
\text { of } 0.45 \% \text { Sodium } \\
\text { Chloride Solution }(\mathrm{N} \\
=\mathbf{3 4 5})\end{array}$ & $\begin{array}{l}\text { Slow Administration } \\
\text { of } 0.9 \% \text { Sodium } \\
\text { Chloride Solution (N } \\
=\mathbf{3 4 9})\end{array}$ \\
\hline \multicolumn{5}{|l|}{ Demographic characteristics } \\
\hline Age - yr & $11.5 \pm 4.06$ & $11.8 \pm 4.26$ & $11.6 \pm 4.09$ & $11.6 \pm 3.89$ \\
\hline Age $<6 \mathrm{yr}$ - no. $(\%)$ & $43(12.5)$ & $42(12.0)$ & $42(12.2)$ & $35(10.0)$ \\
\hline \multicolumn{5}{|l|}{ Race - no./total no. $(\%)^{\dagger}$} \\
\hline White & 235/327 (71.9) & $232 / 328(70.7)$ & 247/331 (74.6) & $243 / 329(73.9)$ \\
\hline Black & $73 / 327(22.3)$ & $82 / 328(25.0)$ & $68 / 331(20.5)$ & $63 / 329(19.1)$ \\
\hline Other & $19 / 327(5.8)$ & $14 / 328(4.3)$ & $16 / 331(4.8)$ & $23 / 329(7.0)$ \\
\hline $\begin{array}{l}\text { Hispanic ethnic group — no./total no. } \\
(\%)^{\dagger}\end{array}$ & 48/331 (14.5) & $62 / 335(18.5)$ & 49/329 (14.9) & $69 / 342(20.2)$ \\
\hline Male sex - no. $(\%)$ & $165(48.0)$ & $164(46.7)$ & $158(45.8)$ & $163(46.7)$ \\
\hline \multicolumn{5}{|l|}{ Diabetes history } \\
\hline $\begin{array}{l}\text { Previous diagnosis of diabetes }- \text { no. } \\
(\%)\end{array}$ & $174(50.6)$ & $182(51.9)$ & $185(53.6)$ & $192(55.0)$ \\
\hline Duration of diabetes $-\mathrm{yr}$ & $4.8 \pm 3.1$ & $5.1 \pm 3.2$ & $4.9 \pm 3.4$ & $4.8 \pm 3.3$ \\
\hline \multicolumn{5}{|c|}{ History of severe hypoglycemia - no./total no. $(\%)$} \\
\hline None & $135 / 170(79.4)$ & $139 / 178(78.1)$ & $144 / 183(78.7)$ & $154 / 188(81.9)$ \\
\hline 1 or 2 episodes & 20/170 (11.8) & 26/178 (14.6) & $29 / 183(15.8)$ & 25/188 (13.3) \\
\hline$>2$ episodes & $15 / 170(8.8)$ & $13 / 178(7.3)$ & $10 / 183(5.5)$ & $9 / 188(4.8)$ \\
\hline \multicolumn{5}{|l|}{$\begin{array}{l}\text { Previous episodes of diabetic } \\
\text { ketoacidosis among patients with } \\
\text { known diabetes - no./total no. (\%) }\end{array}$} \\
\hline None & $42 / 173(24.3)$ & $34 / 181(18.8)$ & $60 / 182(33.0)$ & $58 / 189(30.7)$ \\
\hline 1 or 2 & $74 / 173(42.8)$ & $83 / 181(45.9)$ & $67 / 182(36.8)$ & 77/189 (40.7) \\
\hline$>2$ & $57 / 173(32.9)$ & $64 / 181(35.4)$ & $55 / 182(30.2)$ & $54 / 189(28.6)$ \\
\hline $\begin{array}{l}\text { Glycated hemoglobin level in previous } \\
\mathrm{yr}-\%{ }^{*}\end{array}$ & $10.6 \pm 2.0$ & $10.8 \pm 1.8$ & $10.7 \pm 1.9$ & $10.5 \pm 2.0$ \\
\hline \multicolumn{5}{|l|}{ Mental status at randomization } \\
\hline \multicolumn{5}{|c|}{ Glasgow Coma Scale score at randomization - no. $(\%) \xi$} \\
\hline$<14$ & $7(2.0)$ & $6(1.7)$ & $7(2.0)$ & $8(2.3)$ \\
\hline 14 & $23(6.7)$ & $25(7.1)$ & $26(7.5)$ & $25(7.2)$ \\
\hline 15 & $314(91.3)$ & $320(91.2)$ & $312(90.4)$ & $316(90.5)$ \\
\hline Forward digit-span recall $\mathscr{l}$ & $7.13 \pm 2.39$ & $7.12 \pm 2.28$ & $7.25 \pm 2.07$ & $7.46 \pm 2.37$ \\
\hline Backward digit-span recall ${ }^{q} /$ & $5.54 \pm 2.31$ & $5.47 \pm 2.25$ & $5.51 \pm 2.30$ & $5.76 \pm 2.31$ \\
\hline \multicolumn{5}{|l|}{ Laboratory values at presentation } \\
\hline Glucose $-\mathrm{mg} / \mathrm{dl}$ & $519 \pm 153$ & $524 \pm 150$ & $523 \pm 170$ & $522 \pm 156$ \\
\hline Blood urea nitrogen $-\mathrm{mg} / \mathrm{dl}$ & $17 \pm 8$ & $17 \pm 7$ & $17 \pm 9$ & $17 \pm 7$ \\
\hline
\end{tabular}




\begin{tabular}{|c|c|c|c|c|}
\hline Variable & $\begin{array}{l}\text { Fast Administration } \\
\text { of } 0.45 \% \text { Sodium } \\
\text { Chloride Solution (N } \\
=\mathbf{3 4 4})\end{array}$ & $\begin{array}{l}\text { Fast Administration } \\
\text { of } 0.9 \% \text { Sodium } \\
\text { Chloride Solution (N } \\
=\mathbf{3 5 1})\end{array}$ & $\begin{array}{c}\text { Slow Administration } \\
\text { of 0.45\% Sodium } \\
\text { Chloride Solution (N } \\
=\mathbf{3 4 5} \text { ) }\end{array}$ & $\begin{array}{l}\text { Slow Administration } \\
\text { of } 0.9 \% \text { Sodium } \\
\text { Chloride Solution (N) } \\
=349)\end{array}$ \\
\hline $\mathrm{pH}$ & $7.17 \pm 0.09$ & $7.16 \pm 0.10$ & $7.16 \pm 0.10$ & $7.16 \pm 0.11$ \\
\hline Sodium — mmol/liter & $134 \pm 5$ & $134 \pm 5$ & $134 \pm 5$ & $134 \pm 5$ \\
\hline Bicarbonate $-\mathrm{mmol} /$ liter & $9 \pm 3$ & $9 \pm 3$ & $9 \pm 3$ & $9 \pm 3$ \\
\hline $\mathrm{P}_{\mathrm{co}_{2}}-\mathrm{mm} \mathrm{Hg}$ & $26 \pm 7$ & $26 \pm 7$ & $26 \pm 8$ & $27 \pm 7$ \\
\hline
\end{tabular}

Plus-minus values are means $\pm \mathrm{SD}$. There were no significant differences $(\mathrm{P}<0.05)$ between the groups in any of the comparisons according to Kruskal-Wallis tests for continuous variables and chi-square tests for categorical variables. Patients were permitted to undergo randomization a second time if they had a second episode of diabetic ketoacidosis during the trial, with each randomization considered to be a distinct encounter; therefore, a patient could be represented in more than one group. A total of 132 patients had a second episode of diabetic ketoacidosis and underwent randomization a second time during the trial. Two additional patients who had a third episode of diabetic ketoacidosis inadvertently underwent randomization a third time. Therefore, a total of 1389 distinct episodes of diabetic ketoacidosis were evaluated Data shown are based on encounters. Percentages may not sum to 100 because of rounding. To convert the values for glucose to millimoles per liter, multiply by 0.05551 . To convert the values for blood urea nitrogen to millimoles per liter, multiply by 0.357 .

${ }^{\dagger}$ Race and ethnic group were determined by either patient report or review of medical records.

${ }^{*}$ Data were available for $173,173,177$, and 184 patients in the four listed groups, respectively.

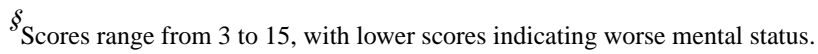

${ }^{9 /}$ Scores range from 0 to 16 , with higher scores indicating better short-term memory. In the digit span test, participants are asked to repeat a sequence of numbers presented orally. In the "forward" task, participants are asked to repeat numbers in order, as presented. In the "backward" task, participants are asked to list the numbers in reverse order. The test stops when participants report the incorrect sequence twice for a given digit-span length. 


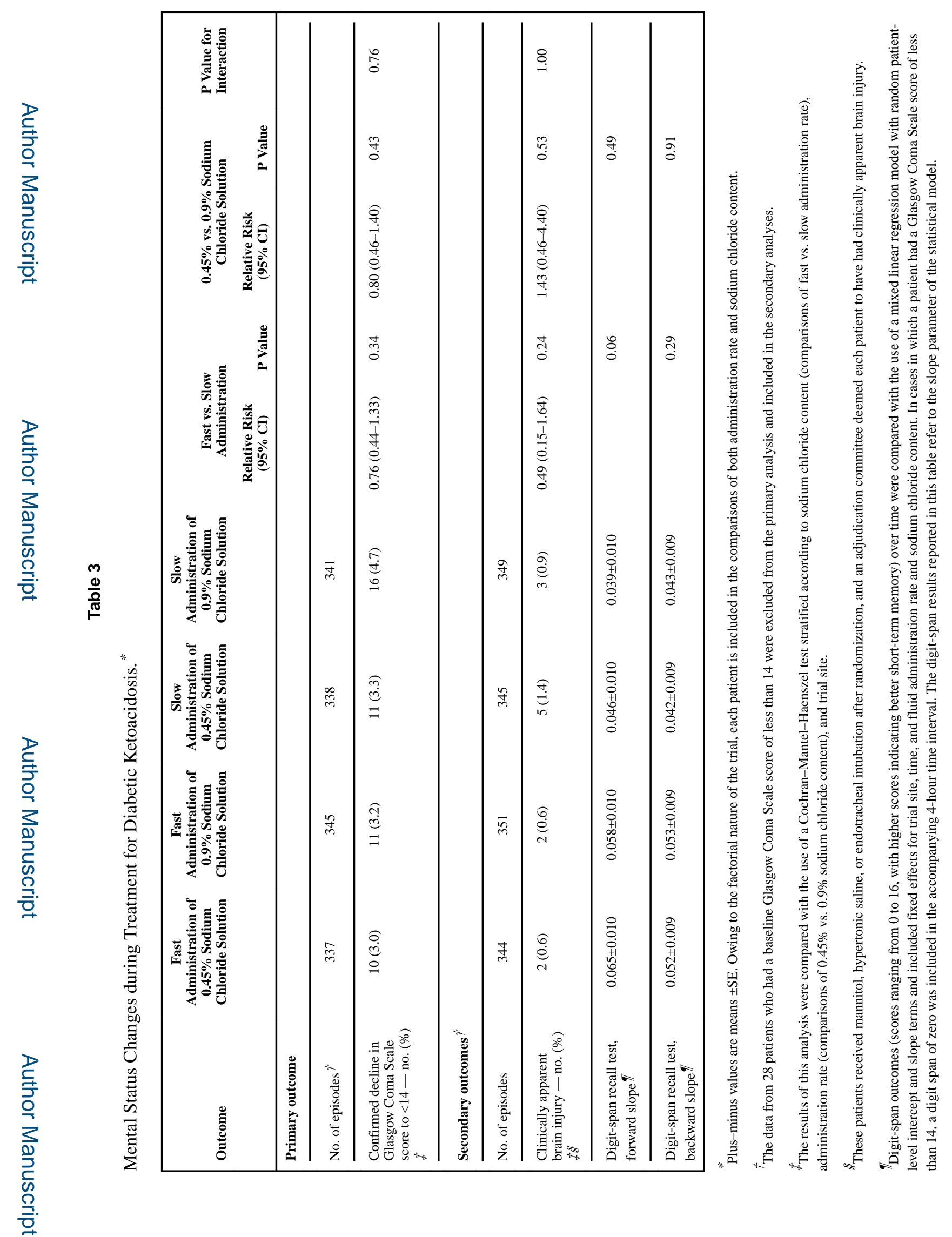

NEngl J Med. Author manuscript; available in PMC 2018 December 14. 
Table 4

Adverse Events. *

\begin{tabular}{|c|c|c|c|c|}
\hline Variable & $\begin{array}{l}\text { Fast Administration } \\
\text { of } 0.45 \% \text { Sodium } \\
\text { Chloride Solution }\end{array}$ & $\begin{array}{l}\text { Fast Administration } \\
\text { of } 0.9 \% \text { Sodium } \\
\text { Chloride Solution }\end{array}$ & $\begin{array}{l}\text { Slow Administration } \\
\text { of } 0.45 \% \text { Sodium } \\
\text { Chloride Solution }\end{array}$ & $\begin{array}{c}\text { Slow Administration } \\
\text { of 0.9\% Sodium } \\
\text { Chloride Solution }\end{array}$ \\
\hline Any adverse event - no. of episodes & 114 & 124 & 118 & 129 \\
\hline Serious adverse event - no. $(\%)$ & $5(4.4)$ & $4(3.2)$ & $11(9.3)$ & $10(7.8)$ \\
\hline \multicolumn{5}{|l|}{ Outcome - no. $(\%)$} \\
\hline $\begin{array}{l}\text { Recovery, with return to baseline } \\
\text { status }\end{array}$ & $102(89.5)$ & $109(87.9)$ & $108(91.5)$ & $118(91.5)$ \\
\hline Recovery with sequelae & $1(0.9)$ & $1(0.8)$ & $1(0.8)$ & $1(0.8)$ \\
\hline Persistence of symptoms & $10(8.8)$ & $14(11.3)$ & $9(7.6)$ & $10(7.8)$ \\
\hline Death & $1(0.9)$ & 0 & 0 & 0 \\
\hline \multicolumn{5}{|l|}{ Severity - no. $(\%)$} \\
\hline Mild & $95(83.3)$ & $96(77.4)$ & $89(75.4)$ & $98(76.0)$ \\
\hline Moderate & $12(10.5)$ & $25(20.2)$ & $22(18.6)$ & $30(23.3)$ \\
\hline Severe & $7(6.1)$ & $3(2.4)$ & $7(5.9)$ & $1(0.8)$ \\
\hline Expected event - no. (\%) & $58(50.9)$ & $56(45.2)$ & $64(54.2)$ & $68(52.7)$ \\
\hline \multicolumn{5}{|l|}{$\begin{array}{l}\text { Common clinical adverse events - } \\
\text { no. }(\%)^{\dagger}\end{array}$} \\
\hline Headache & $29(25.4)$ & $16(12.9)$ & $23(19.5)$ & $28(21.7)$ \\
\hline Oropharyngeal pain & $9(7.9)$ & $11(8.9)$ & $10(8.5)$ & $9(7.0)$ \\
\hline Pyrexia & $8(7.0)$ & $11(8.9)$ & $8(6.8)$ & $10(7.8)$ \\
\hline Abdominal pain & $2(1.8)$ & $11(8.9)$ & $7(5.9)$ & $3(2.3)$ \\
\hline Constipation & $3(2.6)$ & $4(3.2)$ & $2(1.7)$ & $11(8.5)$ \\
\hline
\end{tabular}

* These data were analyzed in the safety population (all patients who underwent randomization and received any trial fluid). Because patients were permitted to undergo randomization twice, a patient could be represented in more than one group. Data are based on patient encounters (i.e., episodes of diabetic ketoacidoses) rather than on individual patients. An episode in which multiple adverse events of the same type occurred is counted once. An episode in which multiple adverse events of different types occurred is counted once for each type of adverse event. In cases in which multiple adverse events occurred in an episode, the most serious adverse event was used to describe seriousness, outcome, and severity.

Common clinical adverse events were events that were reported in six or more patients in any group, excluding adverse events that were counted either as trial outcomes (mental status changes or brain injuries) or as expected nonneurologic adverse events (e.g., hypoglycemia or electrolyte abnormalities) (Table S8 in the Supplementary Appendix). 